\title{
Interior regularity of obstacle problems for nonlinear subelliptic systems with VMO coefficients
}

\section{Guangwei Du* (10) and Fushan Li}

\section{"Correspondence:}

guangwei87@mail.nwpu.edu.cn School of Mathematical Sciences, Qufu Normal University, Qufu, China

\begin{abstract}
This article is concerned with an obstacle problem for nonlinear subelliptic systems of second order with VMO coefficients. It is shown, based on a modification of A-harmonic approximation argument, that the gradient of weak solution to the corresponding obstacle problem belongs to the Morrey space $L_{X, \text { loc }}^{2, \lambda}$.
\end{abstract}

MSC: 35J70; 35B65; 35J50

Keywords: Subelliptic systems; Obstacle problem; VMO; $L_{X, \text { loc }}^{2, \lambda}$-regularity

\section{Introduction}

In this paper we consider weak solutions to an obstacle problem for the following nonlinear subelliptic system in a bounded domain $\Omega$ of Euclidean space $\mathbb{R}^{n}$ :

$$
X_{\alpha}^{*}\left(a_{i j}^{\alpha \beta}(x) X_{\beta} u^{j}\right)=B_{i}(x, u, X u)+X_{\alpha}^{*} g_{i}^{\alpha}(x, u, X u), \quad i=1,2, \ldots, N,
$$

where $X=\left(X_{1}, \ldots, X_{m}\right)(m \leq n)$ is a system of smooth real vector fields satisfying the Hörmander's rank condition, $X_{\alpha}^{*}$ is the formal adjoint of $X_{\alpha}$.

If we set $A(x)=\left\{a_{i j}^{\alpha \beta}(x)\right\}, B=\left(B_{i}\right), g=\left(g_{i}^{\alpha}\right)$, then (1.1) reads

$$
-X^{*}(A(x) X u)=B(x, u, X u)-X^{*} g(x, u, X u) .
$$

Given two vector-valued functions $\psi=\left(\psi^{1}, \ldots, \psi^{N}\right)$ and $\theta=\left(\theta^{1}, \ldots, \theta^{N}\right)$ with $\theta(x) \geq \psi(x)$ a.e. on $\partial \Omega$ (i.e. $\theta^{i}(x) \geq \psi^{i}(x)$ a.e. on $\left.\partial \Omega, i=1,2, \ldots, N\right)$, we define the set

$$
\mathfrak{K}_{\psi}^{\theta}=\left\{v \in S_{X}^{1,2}\left(\Omega, \mathbb{R}^{N}\right): v \geq \psi \text { a.e. in } \Omega, v-\theta \in S_{X, 0}^{1,2}\left(\Omega, \mathbb{R}^{N}\right)\right\} .
$$

Here the functions $\psi$ and $\theta$ are called obstacle and boundary datum, respectively. The function $u \in \mathfrak{K}_{\psi}^{\theta}$ is called a weak solution to the obstacle problem related to (1.1) if

$$
\int_{\Omega} A(x) X u \cdot X \varphi d x \geq \int_{\Omega} B(x, u, X u) \varphi d x+\int_{\Omega} g(x, u, X u) \cdot X \varphi d x
$$

holds for all $\varphi \in C_{0}^{\infty}\left(\Omega, \mathbb{R}^{N}\right)$ with $\varphi+u \geq \psi$ a.e. $x \in \Omega$.

(c) The Author(s) 2018. This article is distributed under the terms of the Creative Commons Attribution 4.0 International License (http://creativecommons.org/licenses/by/4.0/), which permits unrestricted use, distribution, and reproduction in any medium, provided you give appropriate credit to the original author(s) and the source, provide a link to the Creative Commons license, and indicate if changes were made. 
As we know, the uniform ellipticity requirement on coefficients is not sufficient to get the local boundedness of solutions even for one single equation in the Euclidean metric (see [1]). Therefore some additional assumptions on the coefficients is needed to ensure the regularity results. In [2-4], Campanato obtained the $L^{2, \lambda}$-regularity and Hölder continuity for the weak solutions of elliptic systems with continuous coefficients. See also [5-8] for related results.

Since the functions of vanishing mean oscillation (VMO) can have some kind of discontinuities, regularity results under a VMO assumption have been established by many authors; see, for example, [9-12] for elliptic systems, and [13-17] for subelliptic systems constructed by Hörmander's vector fields. Huang in [9] established the gradient estimates in the generalized Morrey spaces of weak solutions to the linear elliptic systems with VMO coefficients. Similar results for the nonlinear elliptic systems were obtained by Daněček and Viszus in [10] and [11]. In [15] and [16] Di Fazio and Fanciullo proved that the local gradient estimates in [9] still hold true for the subelliptic systems structured on Hömander's vector fields. Dong and Niu [14] established the Morrey and Campanato regularity for weak solutions to the nondiagonal subelliptic systems. The direct methods were mainly used to prove the desired results in the papers mentioned above. An important step of this kind of methods is to establish the higher integrability of gradients of weak solutions. These arguments were also used to prove the Morrey regularity and Hölder continuity for weak solutions to the obstacle problems associated with a single elliptic equation with constant coefficients or continuous coefficients; see [18-22].

Recently, another method called $A$-harmonic approximation has been widely applied to prove the optimal partial regularity for nonlinear elliptic systems or subelliptic systems in the Heisenberg group and Carnot groups; see [23-29]. This method is based on Simon's technique of harmonic approximation ([30]) and generalized by Duzaar and Grotowski in [31] in order to deal with partial regularity for nonlinear elliptic systems. The key point is to show that a function which is "approximately harmonic", i.e. a function closes sufficiently to some harmonic function in $L^{2}$. Making use of this method, one can simplify the proof avoiding the proof of a suitable reverse Hölder inequality for the gradient of a weak solution. We also mention that Daněček-John-Stará [32] proved the Morrey space regularity for weak solutions of Stokes systems with VMO coefficients by using a modified $A$-harmonic approximation lemma. Inspired by this work, Yu and Zheng [33] obtained optimal partial regularity for quasilinear elliptic systems with VMO coefficients by a modification of $A$-harmonic approximation argument.

In the present paper we study the interior regularity of weak solutions to the obstacle problem related to the system (1.1) by the technique of $A$-harmonic approximation, which implies that these solutions have the same kind of regularity as the weak solutions of (1.1). Throughout this article, we make the following assumptions.

(H1) The coefficients $a_{i j}^{\alpha \beta}$ are bounded measurable and such that, for some suitable $\lambda>0$ and $\Lambda>0$,

$$
\lambda|\xi|^{2} \leq a_{i j}^{\alpha \beta}(x) \xi_{\alpha}^{i} \xi_{\beta}^{j} \leq \Lambda|\xi|^{2}, \quad x \in \mathbb{R}^{n}, \xi \in \mathbb{R}^{m N}
$$

(H2) The functions $B_{i}, g_{i}^{\alpha}: \mathbb{R}^{n} \times \mathbb{R}^{N} \times \mathbb{R}^{m N} \rightarrow \mathbb{R}$ are both Carathéodory functions and for almost $x \in \Omega$ and all $(u, \xi) \in \mathbb{R}^{N} \times \mathbb{R}^{m N}$, there exists $L>0$ such that

$$
\left|B_{i}(x, u, \xi)\right| \leq f_{i}(x)+L|\xi|^{\gamma_{0}},
$$




$$
\left|g_{i}^{\alpha}(x, u, \xi)\right| \leq f_{i}^{\alpha}(x)+L|\xi|^{\gamma},
$$

where $1 \leq \gamma_{0}<\frac{Q+2}{Q}, 0 \leq \gamma<1$, and

$$
f \in L_{X}^{2 Q /(Q+2), \lambda Q /(Q+2)}\left(\Omega, \mathbb{R}^{N}\right), \quad \tilde{f} \in L_{X}^{2, \lambda}\left(\Omega, \mathbb{R}^{m N}\right), \quad Q-n<\lambda<Q .
$$

Here $Q$ is the homogeneous dimension relative to $\Omega$ and $f=\left(f_{i}\right), \tilde{f}=\left(f_{i}^{\alpha}\right)$.

We are now in the position to state our main result.

Theorem 1.1 Suppose that (H1)-(H2) hold and that $a_{i j}^{\alpha \beta} \in \operatorname{VMO}(\Omega)$ for $i, j=1,2, \ldots, N$, $\alpha, \beta=1,2, \ldots, m$. Let $u \in \mathfrak{K}_{\psi}^{\theta}$ be a weak solution to the obstacle problem for system (1.1) with $X \psi \in L_{X}^{2, \lambda}\left(\Omega, \mathbb{R}^{m N}\right)$, then $X u \in L_{X, \text { loc }}^{2, \lambda}\left(\Omega, \mathbb{R}^{m N}\right)$. Moreover, if $Q-n<\lambda<2$ then $u \in$ $C_{X}^{0,(2-\lambda) / 2}\left(\Omega, \mathbb{R}^{N}\right)$.

The paper is organized as follows. In the next section we recall some concepts and facts associated to Carnot-Carathéodory spaces and give the proof of the modified $A$-harmonic approximation lemma for vector fields. In Sect. 3, we consider the following linear subelliptic system with VMO coefficients:

$$
X^{*}(A(x) X u)=X^{*}(A(x) X \psi)
$$

and we prove a comparison principle and a Morrey type estimate for weak solutions of the above system by a modification of $A$-harmonic approximation argument. Section 4 is devoted to the proofs of Theorem 1.1. On the basis of the Morrey type estimate established for linear subelliptic system, we can first prove the $L_{X, \text { loc }}^{2, \lambda}$-regularity for weak solutions of the obstacle problems and then interior Hölder continuity is obtained by virtue of the equivalence between the Campanato space and the Hölder continuity function space (see $[34,35])$.

In what follows, we use $c$ to denote a positive constant that may vary from line to line.

\section{Some notations and preliminaries}

Let

$$
X_{\alpha}=\sum_{k=1}^{n} b_{\alpha k} \frac{\partial}{\partial x_{k}}, \quad b_{\alpha k} \in C^{\infty}, \alpha=1,2, \ldots, m
$$

be a family of vector fields in $\mathbb{R}^{n}$ satisfying Hörmander's condition ([36]):

$$
\operatorname{rank}\left(\operatorname{Lie}\left\{X_{1}, \ldots, X_{m}\right\}\right)=n
$$

We consider $X_{\alpha}$ as a first order differential operator acting on $u \in \operatorname{Lip}\left(\mathbb{R}^{n}\right)$ defined as

$$
X_{\alpha} u(x)=\left\langle X_{\alpha}(x), \nabla u(x)\right\rangle, \quad \alpha=1,2, \ldots, m .
$$

We denote by $X u=\left(X_{1} u, \ldots, X_{m} u\right)$ the gradient of $u$ and hence $|X u(x)|=\left(\sum_{\alpha=1}^{m} \mid X_{\alpha} u(x)^{2}\right)^{\frac{1}{2}}$. An absolutely continuous curve $\gamma:[a, b] \rightarrow \mathbb{R}^{n}$ is said to be admissible if

$$
\gamma^{\prime}(t)=\sum_{\alpha=1}^{m} c_{\alpha}(t) X_{\alpha}(\gamma(t)), \quad \text { a.e. } t \in[a, b]
$$


for some functions $c_{\alpha}(t)$ satisfying $\sum_{\alpha=1}^{m} c_{\alpha}(t)^{2} \leq 1$. The Carnot-Carathéodory distance $d(x, y)$ generated by $X$ is defined by

$$
d(x, y)=\inf \{T>0: \text { there is an admissible curve } \gamma, \gamma(0)=x, \gamma(T)=y\} .
$$

For $x \in \mathbb{R}^{n}$ and $R>0$ we let

$$
B_{R}(x)=B(x, R)=\left\{y \in \mathbb{R}^{n}: d(y, x)<R\right\} .
$$

In what follows, if $\sigma>0$ and $B=B(x, R)$ we write $\sigma B$ to indicate $B(x, \sigma R)$. Furthermore, if $E \subset \mathbb{R}^{n}$ is a Lebesgue measurable set with Lebesgue measure $|E|$, we set $u_{E}=f_{E} u d x$ the integral average of $u$ on $E$.

In [37], it was proved that for every connected $K \subset \Omega$ there exist constants $C_{1}, C_{2}>0$ and $0<\lambda<1$ such that

$$
C_{1}|x-y| \leq d(x, y) \leq C_{2}|x-y|^{\lambda}, \quad x, y \in K .
$$

Moreover, there are $R_{d}>0$ and $C_{d} \geq 1$ such that, for any $x \in K$ and $R \leq R_{d}$,

$$
|B(x, 2 R)| \leq C_{d}|B(x, R)| .
$$

Property (2.1) is the so-called "doubling condition" which is assumed to hold on the spaces of homogeneous type. The best constant $C_{d}$ in (2.1) is called the doubling constant. We call that $Q=\log _{2} C_{d}$ is the homogeneous dimension relative to $\Omega$. As a consequence of (2.1), we have

$$
\left|B_{t R}\right| \geq C_{d}^{-2} t^{Q}\left|B_{R}\right|, \quad \forall R \leq R_{d}, t \in(0,1) .
$$

We now introduce the relevant Sobolev spaces. Given $1 \leq p<\infty$, the Sobolev space $S_{X}^{1, p}\left(\Omega, \mathbb{R}^{N}\right)$ is the Banach space

$$
S_{X}^{1, p}\left(\Omega, \mathbb{R}^{N}\right)=\left\{u \in L^{p}\left(\Omega, \mathbb{R}^{N}\right): X_{\alpha} u \in L^{p}\left(\Omega, \mathbb{R}^{N}\right), \alpha=1,2, \ldots, m\right\}
$$

endowed with the norm

$$
\|u\|_{S_{X}^{1, p}\left(\Omega, \mathbb{R}^{N}\right)}=\|u\|_{L^{p}\left(\Omega, \mathbb{R}^{N}\right)}+\sum_{\alpha=1}^{m}\left\|X_{\alpha} u\right\|_{L^{p}\left(\Omega, \mathbb{R}^{N}\right)} .
$$

Here, $X_{\alpha} u$ is the distributional derivative of $u \in L_{\text {loc }}^{1}\left(\Omega, \mathbb{R}^{N}\right)$ defined by

$$
\int_{\Omega} X_{\alpha} u \cdot \phi d x=\int_{\Omega} u \cdot X_{\alpha}^{*} \phi d x, \quad \forall \phi \in C_{0}^{\infty}\left(\Omega, \mathbb{R}^{N}\right),
$$

where

$$
X_{\alpha}^{*}=-\sum_{k=1}^{n} \frac{\partial}{\partial x_{k}}\left(b_{\alpha k} \cdot\right)
$$


is the formal adjoint of $X_{\alpha}$, not necessarily a vector field in general. The space $S_{X, 0}^{1, p}\left(\Omega, \mathbb{R}^{N}\right)$ is defined as the completion of $C_{0}^{\infty}\left(\Omega, \mathbb{R}^{N}\right)$ under the norm $\|\cdot\|_{S_{X}^{1, p}\left(\Omega, \mathbb{R}^{N}\right)}$.

In addition, we also need the following Sobolev inequalities for vector fields.

Theorem 2.1 ([38,39]) For every compact set $K \subset \Omega$, there exist constants $C>0$ and $\bar{R}>0$ such that, for any metric ball $B=B\left(x_{0}, R\right)$ with $x_{0} \in K$ and $0<R \leq \bar{R}$, for any $f \in S_{X}^{1, p}\left(B_{R}\right)$,

$$
\left(f_{B_{R}}\left|f-f_{R}\right|^{\kappa p} d x\right)^{\frac{1}{\kappa p}} \leq C R\left(f_{B_{R}}|X f|^{p} d x\right)^{\frac{1}{p}},
$$

where $1 \leq \kappa \leq Q /(Q-p)$, if $1 \leq p<Q ; 1 \leq \kappa<\infty$, if $p \geq Q$. Moreover,

$$
\left(f_{B_{R}}|f|^{\kappa p} d x\right)^{\frac{1}{\kappa p}} \leq C R\left(f_{B_{R}}|X f|^{p} d x\right)^{\frac{1}{p}}
$$

whenever $f \in S_{X, 0}^{1, p}\left(B_{R}\right)$.

Now we define the Morrey spaces, the Campanato spaces, VMO and the Hölder spaces with respect to the Carnot-Carathéodory metric. To simplify our description, we introduce the following notation:

$$
\Omega(x, R)=\Omega \cap B(x, R), \quad f_{x, R}=\frac{1}{|\Omega(x, R)|} \int_{\Omega(x, R)} f(y) d y,
$$

and

$$
d_{0}=\min \left\{\operatorname{diam} \Omega, R_{d}\right\}
$$

Definition 2.2 For $1<p<\infty$ and $\lambda \leq Q$, we say that $f \in L_{\text {loc }}^{p}\left(\Omega, \mathbb{R}^{N}\right)$ belongs to the Morrey space $L_{X}^{p, \lambda}\left(\Omega, \mathbb{R}^{N}\right)$ if

$$
\|f\|_{L_{X}^{p, \lambda}\left(\Omega, \mathbb{R}^{N}\right)}=\sup _{x \in \Omega, 0<\rho<d_{0}}\left(\frac{\rho^{\lambda}}{|\Omega(x, \rho)|} \int_{\Omega(x, \rho)}|f(y)|^{p} d y\right)^{\frac{1}{p}}<\infty ;
$$

$f \in L_{\text {loc }}^{p}\left(\Omega, \mathbb{R}^{N}\right)$ belongs to the Campanato space $\mathcal{L}_{X}^{p, \lambda}\left(\Omega, \mathbb{R}^{N}\right)$ if

$$
\|f\|_{\mathcal{L}_{X}^{p, \lambda}\left(\Omega, \mathbb{R}^{N}\right)}=\sup _{x \in \Omega, 0<\rho<d_{0}}\left(\frac{\rho^{\lambda}}{|\Omega(x, \rho)|} \int_{\Omega(x, \rho)}\left|f(y)-f_{x, \rho}\right|^{p} d y\right)^{\frac{1}{p}}<\infty .
$$

Definition 2.3 For $\alpha \in(0,1)$, the Hölder space $C_{X}^{0, \alpha}\left(\bar{\Omega}, \mathbb{R}^{N}\right)$ is the collection of functions $f: \bar{\Omega} \rightarrow \mathbb{R}^{N}$ satisfying

$$
\|f\|_{C_{X}^{0, \alpha}\left(\bar{\Omega}, \mathbb{R}^{N}\right)}=\sup _{\Omega}|f|+\sup _{\bar{\Omega}} \frac{|f(x)-f(y)|}{d(x, y)^{\alpha}}<\infty .
$$

We say that $f$ is locally Hölder continuous, i.e. $f \in C_{X}^{0, \alpha}\left(\Omega, \mathbb{R}^{N}\right)$, if $f \in C_{X}^{0, \alpha}\left(K, \mathbb{R}^{N}\right)$ for every compact set $K \subset \Omega$. 
Definition 2.4 We say that $f \in L_{\text {loc }}^{1}\left(\Omega, \mathbb{R}^{N}\right)$ belongs to $\operatorname{BMO}\left(\Omega, \mathbb{R}^{N}\right)$ if

$$
\|f\|_{*}=\sup _{x \in \Omega, 0<\rho<d_{0}} \frac{1}{|\Omega(x, \rho)|} \int_{\Omega(x, \rho)}\left|f(y)-f_{x, \rho}\right| d y<\infty
$$

$f$ belongs to $\operatorname{VMO}\left(\Omega, \mathbb{R}^{N}\right)$ if $f \in \operatorname{BMO}\left(\Omega, \mathbb{R}^{N}\right)$ and

$$
\eta_{r}(f)=\sup _{x \in \Omega, 0<\rho<r} \frac{1}{|\Omega(x, \rho)|} \int_{\Omega(x, \rho)}\left|f(y)-f_{x, \rho}\right| d y \rightarrow 0, \quad r \rightarrow 0 .
$$

The integral characterization for a Hölder continuous function was shown in [35] and [34].

Lemma 2.5 If $-p<\lambda<0$, then $\mathcal{L}_{X}^{p, \lambda}\left(\Omega, \mathbb{R}^{N}\right) \simeq C_{X}^{0, \alpha}\left(\Omega, \mathbb{R}^{N}\right), \alpha=-\frac{\lambda}{p}$.

\section{Morrey type estimate for a subelliptic system}

In this section we will prove by the modified $A$-harmonic approximation technique a Morrey type estimate for the subelliptic system

$$
X^{*}(A(x) X u)=X^{*}(A(x) X \psi)
$$

Let us first recall that a function $h \in S_{X}^{1,2}\left(\Omega, \mathbb{R}^{N}\right)$ is called $A$-harmonic for $A \in \operatorname{Bil}\left(\mathbb{R}^{m N}\right)$ if $h$ satisfies

$$
\int_{\Omega} A(X h, X \varphi) d x=0, \quad \forall \varphi \in C_{0}^{1}\left(\Omega, \mathbb{R}^{N}\right)
$$

We cite the $A$-harmonic approximation lemma for vector fields as follows ([24, 31]).

Lemma 3.1 Consider fixed positive $\lambda$ and $\Lambda$, and $m, N \in \mathbb{N}$ with $m \geq 2$. Then for any given $\varepsilon>0$ there exists $\delta=\delta(m, N, \lambda, \Lambda, \varepsilon)$ with the following property: for any $A \in \operatorname{Bil}\left(\mathbb{R}^{m N}\right)$ satisfying

$$
A(\xi, \xi) \geq \lambda|\xi|^{2}, \quad \text { for all } \xi \in \mathbb{R}^{m N}
$$

and

$$
A(\xi, \tilde{\xi}) \leq \Lambda|\xi||\tilde{\xi}|, \quad \text { for all } \xi, \tilde{\xi} \in \mathbb{R}^{m N}
$$

for any $g \in S_{X}^{1,2}\left(B_{\rho}\left(x_{0}\right), \mathbb{R}^{N}\right)$ (for some $\rho>0, x_{0} \in \mathbb{R}^{n}$ ) satisfying

$$
f_{B_{\rho}\left(x_{0}\right)}|X g|^{2} d x \leq 1
$$

and

$$
\left|f_{B_{\rho}\left(x_{0}\right)} A(X g, X \varphi) d x\right| \leq \delta \sup _{B_{\rho}\left(x_{0}\right)}|X \varphi|, \quad \forall \varphi \in C_{0}^{\infty}\left(B_{\rho}\left(x_{0}\right), \mathbb{R}^{N}\right)
$$


there exists an A-harmonic function $h \in S_{X}^{1,2}\left(B_{\rho}\left(x_{0}\right), \mathbb{R}^{N}\right)$ such that

$$
f_{B_{\rho}\left(x_{0}\right)}|X h|^{2} d x \leq 1 \text { and } \frac{1}{\rho^{2}} f_{B_{\rho}\left(x_{0}\right)}|g-h|^{2} d x \leq \varepsilon .
$$

Similarly to [32] and [33], we can prove the following modification of the $A$-harmonic approximation lemma.

Lemma 3.2 Let $0<\lambda<\Lambda$ and $m \in \mathbb{N}$ with $m \geq 2$ be fixed. Then, for any $\varepsilon>0$, there exists a constant $k=k(m, N, \lambda, \Lambda, \varepsilon)$ such that the following holds: for any $A \in \operatorname{Bil}\left(\mathbb{R}^{m N}\right)$ satisfying conditions (3.2), (3.3) and for any $u \in S_{X}^{1,2}\left(B_{\rho}\left(x_{0}\right), \mathbb{R}^{N}\right)$, there exists an $A$-harmonic function $h \in S_{X}^{1,2}\left(B_{\rho}\left(x_{0}\right), \mathbb{R}^{N}\right)$ such that

$$
\int_{B_{\rho}\left(x_{0}\right)}|X h|^{2} d x \leq \int_{B_{\rho}\left(x_{0}\right)}|X u|^{2} d x
$$

and, moreover, there exists $\varphi \in C_{0}^{\infty}\left(B_{\rho}\left(x_{0}\right), \mathbb{R}^{N}\right)$ such that

$$
\|X \varphi\|_{L^{\infty}\left(B_{\rho}\left(x_{0}\right), \mathbb{R}^{N}\right)} \leq \frac{1}{\rho}
$$

and

$$
\begin{aligned}
& \int_{B_{\rho}\left(x_{0}\right)}|u-h|^{2} d x \\
& \quad \leq \varepsilon \rho^{2} \int_{B_{\rho}\left(x_{0}\right)}|X u|^{2} d x+k(\varepsilon)\left[\frac{\rho^{4}}{\left|B_{\rho}\left(x_{0}\right)\right|}\left(\int_{B_{\rho}\left(x_{0}\right)} A(X u, X \varphi) d x\right)^{2}\right] .
\end{aligned}
$$

Proof For any given $\varepsilon>0$ and $u \in S_{X}^{1,2}\left(B_{\rho}\left(x_{0}\right), \mathbb{R}^{N}\right)$, we take $\delta(\varepsilon)$ as in the above Lemma 3.1 and set

$$
g=\left(f_{B_{\rho}\left(x_{0}\right)}|X u|^{2} d x\right)^{-\frac{1}{2}} u .
$$

Then (3.4) holds. Assume that for $g$ the inequality (3.5) is true. From Lemma 3.1, there is an $A$-harmonic function $w$ satisfying

$$
f_{B_{\rho}\left(x_{0}\right)}|X w|^{2} d x \leq 1, \quad \frac{1}{\rho^{2}} f_{B_{\rho}\left(x_{0}\right)}|w-g|^{2} d x \leq \varepsilon
$$

and thus the function $h=\left(f_{B_{\rho}\left(x_{0}\right)}|X u|^{2} d x\right)^{\frac{1}{2}} w$ satisfies (3.6). Moreover, we have

$$
|u-h|^{2}=f_{B_{\rho}\left(x_{0}\right)}|X u|^{2} d x \cdot|g-w|^{2},
$$

which implies

$$
\int_{B_{\rho}\left(x_{0}\right)}|u-h|^{2} d x \leq \int_{B_{\rho}\left(x_{0}\right)}|X u|^{2} d x \cdot f_{B_{\rho}\left(x_{0}\right)}|g-w|^{2} d x \leq \varepsilon \rho^{2} \int_{B_{\rho}\left(x_{0}\right)}|X u|^{2} d x .
$$


If, vice versa, there is a nonconstant function $\tilde{\varphi} \in C_{0}^{\infty}\left(B_{\rho}\left(x_{0}\right), \mathbb{R}^{N}\right)$ such that

$$
\left|f_{B_{\rho}\left(x_{0}\right)} A(X g, X \tilde{\varphi}) d x\right|>\delta(\varepsilon) \sup _{B_{\rho}\left(x_{0}\right)}|X \tilde{\varphi}|
$$

Setting $\varphi=\frac{\tilde{\varphi}}{\rho \sup _{B \rho}\left(x_{0}\right)|X \tilde{\varphi}|}$, it follows that

$$
\frac{\rho}{\delta(\varepsilon)}\left|f_{B_{\rho}\left(x_{0}\right)} A(X g, X \varphi) d x\right|>1
$$

We now take $h=u_{\rho}$. Using the Poincaré inequality and the fact that $X g=$ $\left(f_{B_{\rho}\left(x_{0}\right)}|X u|^{2} d x\right)^{-\frac{1}{2}} X u$ we deduce

$$
\begin{aligned}
\int_{B_{\rho}\left(x_{0}\right)}|u-h|^{2} d x & =\int_{B_{\rho}\left(x_{0}\right)}\left|u-u_{\rho}\right|^{2} d x \\
& \leq c \rho^{2} \int_{B_{\rho}\left(x_{0}\right)}|X u|^{2} d x \\
& \leq \frac{c \rho^{4}}{\delta^{2}(\varepsilon)}\left|f_{B_{\rho}\left(x_{0}\right)} A(X g, X \varphi) d x\right|^{2} \int_{B_{\rho}\left(x_{0}\right)}|X u|^{2} d x \\
& \leq \frac{c \rho^{4}\left|B_{\rho}\left(x_{0}\right)\right|}{\delta^{2}(\varepsilon)}\left|f_{B_{\rho}\left(x_{0}\right)} A(X u, X \varphi) d x\right|^{2} \\
& \leq \frac{c \rho^{4}}{\delta^{2}(\varepsilon)\left|B_{\rho}\left(x_{0}\right)\right|}\left|\int_{B_{\rho}\left(x_{0}\right)} A(X u, X \varphi) d x\right|^{2}
\end{aligned}
$$

Combining (3.9) and (3.10) and taking $k(\varepsilon)=\frac{c}{\delta^{2}(\varepsilon)}$ complete the proof.

Now we are in a position to establish the Morrey type estimate for gradient of weak solution to (3.1) based on Lemma 3.2.

Lemma 3.3 Suppose that $A(x)$ satisfies $(\mathrm{H} 1)$ and $u \in S_{X, \mathrm{loc}}^{1,2}\left(\Omega, \mathbb{R}^{N}\right)$ is a weak solution to the system (3.1), i.e.,

$$
\int_{\Omega} A(x) X u \cdot X \varphi d x=\int_{\Omega} A(x) X \psi \cdot X \varphi d x, \quad \forall \varphi \in C_{0}^{\infty}\left(\Omega, \mathbb{R}^{N}\right)
$$

Then for any $x_{0} \in \Omega$ there exists a constant $c>0$ such that, for all $B_{\rho}\left(x_{0}\right) \subset B_{R}\left(x_{0}\right) \subset \Omega$, $R<R_{d}$

$$
\int_{B_{\rho}\left(x_{0}\right)}|X u|^{2} d x \leq c\left[\left(\frac{\rho}{R}\right)^{Q}+\varepsilon+\eta_{R}(A)\right] \int_{B_{R}\left(x_{0}\right)}|X u|^{2} d x+c \int_{B_{R}\left(x_{0}\right)}|X \psi|^{2} d x
$$

Proof For fixed $x_{0} \in \Omega$ and $0<R<R_{d}$, denote $B_{R}:=B_{R}\left(x_{0}\right)$. Let $\eta$ be a cut-off function on $B_{R}$ relative to $B_{\rho}$, i.e. $\eta \in C_{0}^{\infty}\left(B_{R}, \mathbb{R}^{N}\right)$ and satisfies

$$
0 \leq \eta(x) \leq 1, \quad \eta(x)=1 \quad \text { in } B_{\rho}, \quad|X \eta(x)| \leq \frac{c}{R-\rho} .
$$


Taking the function $\varphi=\eta^{2}\left(u-u_{R}\right)$ as a test function, it follows that

$$
\begin{aligned}
\int_{B_{R}} & \eta^{2} A(x) X u \cdot X u d x \\
= & -2 \int_{B_{R}} A(x) \eta\left(u-u_{R}\right) X u \cdot X \eta d x+\int_{B_{R}} \eta^{2} A(x) X \psi \cdot X u d x \\
& +2 \int_{B_{R}} A(x) \eta\left(u-u_{R}\right) X \psi \cdot X \eta d x .
\end{aligned}
$$

From (H1) and Young's inequality

$$
\begin{aligned}
\lambda \int_{B_{R}} \eta^{2}|X u|^{2} d x \leq & 2 \Lambda \int_{B_{R}}|\eta X u|\left|u-u_{R}\right||X \eta| d x \\
& +\Lambda \int_{B_{R}}|\eta X \psi||\eta X u| d x+2 \Lambda \int_{B_{R}}|\eta X \psi|\left|u-u_{R}\right||X \eta| d x \\
\leq & \varepsilon \int_{B_{R}} \eta^{2}|X u|^{2} d x+\frac{c_{\varepsilon}}{(R-\rho)^{2}} \int_{B_{R}}\left|u-u_{R}\right|^{2} d x+c_{\varepsilon} \int_{B_{R}}|X \psi|^{2} d x
\end{aligned}
$$

Choosing $\varepsilon<\lambda$, it follows that

$$
\int_{B_{\rho}}|X u|^{2} d x \leq \frac{c}{(R-\rho)^{2}} \int_{B_{R}}\left|u-u_{R}\right|^{2} d x+c \int_{B_{R}}|X \psi|^{2} d x
$$

Next we define $A_{R}=f_{B_{R}} A(x) d x$. By Lemma 3.2, there exists an $A_{R}$-harmonic function $h \in S_{X}^{1,2}\left(B_{R}, \mathbb{R}^{N}\right)$ such that (3.6)-(3.8) hold. Moreover, by standard results of the subelliptic system with constant coefficients (see for example [34]), we have

$$
\int_{B_{\rho}}|X h|^{2} d x \leq c\left(\frac{\rho}{R}\right)^{Q} \int_{B_{R}}|X h|^{2} d x, \quad \forall 0<\rho \leq R
$$

Therefore, from (3.12) and (3.6) it follows that for any $0<\rho<R / 2$

$$
\begin{aligned}
& \int_{B_{\rho}}|X u|^{2} d x \\
& \quad \leq \frac{c}{\rho^{2}} \int_{B_{2 \rho}}\left|u-u_{2 \rho}\right|^{2} d x+c \int_{B_{2 \rho}}|X \psi|^{2} d x \\
& \leq \frac{c}{\rho^{2}}\left(\int_{B_{2 \rho}}\left|u-u_{2 \rho}-\left(h-h_{2 \rho}\right)\right|^{2} d x+\int_{B_{2 \rho}}\left|h-h_{2 \rho}\right|^{2} d x\right)+c \int_{B_{2 \rho}}|X \psi|^{2} d x \\
& \quad \leq \frac{c}{\rho^{2}} \int_{B_{2 \rho}}|u-h|^{2} d x+c \int_{B_{2 \rho}}|X h|^{2} d x+c \int_{B_{2 \rho}}|X \psi|^{2} d x \\
& \leq \frac{c}{\rho^{2}} \int_{B_{2 \rho}}|u-h|^{2} d x+c\left(\frac{\rho}{R}\right)^{Q} \int_{B_{R}}|X h|^{2} d x+c \int_{B_{R}}|X \psi|^{2} d x \\
& \leq \frac{c}{\rho^{2}} \int_{B_{2 \rho}}|u-h|^{2} d x+c\left(\frac{\rho}{R}\right)^{Q} \int_{B_{R}}|X u|^{2} d x+c \int_{B_{R}}|X \psi|^{2} d x .
\end{aligned}
$$


For the first term in the right-hand side, we have from Lemma 3.2

$$
\begin{aligned}
\frac{c}{\rho^{2}} \int_{B_{2 \rho}}|u-h|^{2} d x & \leq c \varepsilon \int_{B_{2 \rho}}|X u|^{2} d x+c k(\varepsilon) \frac{\rho^{2}}{\left|B_{2 \rho}\right|}\left(\int_{B_{2 \rho}} A_{R} X u \cdot X \varphi d x\right)^{2} \\
& \leq c \varepsilon \int_{B_{R}}|X u|^{2} d x+c_{\varepsilon} \frac{\rho^{2}}{\left|B_{R}\right|}\left(\int_{B_{R}} A_{R} X u \cdot X \varphi d x\right)^{2}
\end{aligned}
$$

where $\varphi \in C_{0}^{\infty}\left(B_{2 \rho}, \mathbb{R}^{N}\right)$ satisfies $\|X \varphi\|_{L^{\infty}\left(B_{2 \rho}, \mathbb{R}^{N}\right)} \leq \frac{1}{R}<\frac{1}{2 \rho}$. Since $u$ is a weak solution to (3.1), it follows that

$$
\begin{aligned}
\left(\int_{B_{R}} A_{R} X u \cdot X \varphi d x\right)^{2} & =\left(\int_{B_{R}}\left(A_{R}-A\right) X u \cdot X \varphi d x+\int_{B_{R}} A X u \cdot X \varphi d x\right)^{2} \\
& \leq 2\left(\int_{B_{R}}\left(A_{R}-A\right) X u \cdot X \varphi d x\right)^{2}+2\left(\int_{B_{R}} A X u \cdot X \varphi d x\right)^{2} \\
& :=I_{1}+I_{2} .
\end{aligned}
$$

From Hölder's inequality, using (H1), we have

$$
\begin{aligned}
I_{1} & \leq \frac{1}{2 \rho^{2}} \int_{B_{R}}|X u|^{2} d x \cdot \int_{B_{R}}\left|A_{R}-A\right|^{2} d x \\
& \leq \frac{\Lambda\left|B_{R}\right|}{\rho^{2}} \frac{1}{\left|B_{R}\right|} \int_{B_{R}}\left|A_{R}-A\right| d x \cdot \int_{B_{R}}|X u|^{2} d x \\
& \leq \frac{\Lambda\left|B_{R}\right|}{\rho^{2}} \eta_{R}(A) \int_{B_{R}}|X u|^{2} d x
\end{aligned}
$$

and

$$
I_{2} \leq \frac{\Lambda^{2}}{2 \rho^{2}}\left(\int_{B_{R}}|X \psi| d x\right)^{2} \leq \frac{\Lambda^{2}\left|B_{R}\right|}{2 \rho^{2}} \int_{B_{R}}|X \psi|^{2} d x
$$

Hence

$$
\left(\int_{B_{R}} A_{R} X u \cdot X \varphi d x\right)^{2} \leq \frac{c\left|B_{R}\right|}{\rho^{2}}\left[\eta_{R}(A) \int_{B_{R}}|X u|^{2} d x+\int_{B_{R}}|X \psi|^{2} d x\right] .
$$

Combining (3.15), (3.14) and (3.13), we have, for any $0<\rho<R / 2$,

$$
\int_{B_{\rho}}|X u|^{2} d x \leq c\left[\left(\frac{\rho}{R}\right)^{Q}+\varepsilon+\eta_{R}(A)\right] \int_{B_{R}}|X u|^{2} d x+c \int_{B_{R}}|X \psi|^{2} d x .
$$

For $R / 2 \leq \rho \leq R$, obviously

$$
\int_{B_{\rho}}|X u|^{2} d x \leq \int_{B_{R}}|X u|^{2} d x \leq 2^{Q}\left(\frac{\rho}{R}\right)^{Q} \int_{B_{R}}|X u|^{2} d x
$$

A combination of these two cases leads to (3.11) for $0<\rho \leq R$.

We end this section with a comparison principle for system (3.1). 
Lemma 3.4 Suppose that $u, \psi \in S_{X}^{1,2}\left(B_{R}, \mathbb{R}^{N}\right)$ satisfy

$$
X^{*}(A(x) X u)=X^{*}(A(x) X \psi)
$$

where $A(x)$ satisfies $(\mathrm{H} 1)$. If $\psi \leq u$ on $\partial B_{R}$, then $\psi \leq u$ a.e. in $B_{R}$.

Proof For any $\varphi \in C_{0}^{\infty}\left(B_{R}, \mathbb{R}^{N}\right)$ we have

$$
\int_{B_{R}} A(x) X u \cdot X \varphi d x=\int_{B_{R}} A(x) X \psi \cdot X \varphi d x
$$

Set $u_{+}=\max \{u, 0\}$. Since $\psi \leq u$ on $\partial B_{R}$, we conclude that (see [40, Lemma 6]) $(\psi-u)_{+} \in$ $S_{X, 0}^{1,2}\left(B_{R}, \mathbb{R}^{N}\right)$ and

$$
X(\psi-u)_{+}= \begin{cases}X(\psi-u), & \psi>u \\ 0, & \psi \leq u\end{cases}
$$

Choosing $\varphi=(\psi-u)_{+}$in (3.16) gives

$$
\int_{B_{R}} A(x) X(\psi-u) \cdot X(\psi-u)_{+} d x=0
$$

which implies

$$
\int_{B_{R} \cap\{\psi>u\}} A(x) X(\psi-u) \cdot X(\psi-u) d x=0
$$

From (H1) we have

$$
\begin{aligned}
\int_{B_{R}}\left|X(\psi-u)_{+}\right|^{2} d x & =\int_{B_{R} \cap\{\psi>u\}}|X(\psi-u)|^{2} d x \\
& \leq \frac{1}{\lambda} \int_{B_{R} \cap\{\psi>u\}} A(x) X(\psi-u) \cdot X(\psi-u) d x=0 .
\end{aligned}
$$

Thus from Poincaré inequality, we obtain

$$
\int_{B_{R}}\left|(\psi-u)_{+}\right|^{2} d x \leq c R^{2} \int_{B_{R}}\left|X(\psi-u)_{+}\right|^{2} d x=0
$$

which implies $(\psi-u)_{+}=0$, or $\psi \leq u$ a.e. in $B_{R}$. The proof is complete.

\section{Proof of main result}

In this section we are going to prove our main result. To this end, we need a generalized iteration lemma, which can be found in [9, Proposition 2.1].

Lemma 4.1 Let $H$ be a nonnegative almost increasing function on the interval $[0, T]$ and $F$ a positive function on $(0, T]$. Suppose that 
(1) for any $0<\rho \leq R \leq T$, there exist $A, B$, $\varepsilon$ and $a>0$ such that

$$
H(\rho) \leq\left(A\left(\frac{\rho}{R}\right)^{a}+\varepsilon\right) H(R)+B F(R)
$$

(2) there exists $\tau \in(0, a)$ such that $\frac{\rho^{\tau}}{F(\rho)}$ is almost increasing in $(0, T]$. Then there exist positive constants $\varepsilon_{0}$ and $C$ such that, for any $0 \leq \varepsilon \leq \varepsilon_{0}$,

$$
H(\rho) \leq C \frac{F(\rho)}{F(R)} H(R)+C B \cdot F(\rho), \quad 0<\rho \leq R \leq T,
$$

where $\varepsilon_{0}$ depends only on $A, a$ and $\tau$.

Proof of Theorem 1.1 Let $B_{R}=B\left(x_{0}, R\right) \subset \subset \Omega$ be an arbitrary ball around $x_{0}$ of radius $R$ and let $u \in \mathfrak{K}_{\psi}^{\theta}$ be a weak solution to the obstacle problem related to (1.1). In $B_{R}$ we split $u$ as $u=w+(u-w)$, where $w \in S_{X}^{1,2}\left(B_{R}, \mathbb{R}^{N}\right)$ is the weak solution to the following system:

$$
\begin{cases}X^{*}(A(x) X w)=X^{*}(A(x) X \psi) & \text { in } B_{R} \\ w=u & \text { on } \partial B_{R}\end{cases}
$$

Since $w=u \geq \psi$ a.e. on $\partial B_{R}$, it follows from Lemma 3.4 that $w \geq \psi$ a.e. in $B_{R}$.

By the definition of weak solutions, we have

$$
\int_{B_{R}} A(x) X w \cdot X(w-u) d x=\int_{B_{R}} A(x) X \psi \cdot X(w-u) d x
$$

From (H1) and Young's inequality one gets

$$
\begin{aligned}
\lambda \int_{B_{R}}|X w|^{2} d x & \leq \int_{B_{R}} A(x) X w \cdot X w d x \\
& \leq \Lambda \int_{B_{R}}|X w||X u| d x+\Lambda \int_{B_{R}}|X \psi||X w-X u| d x \\
& \leq \varepsilon \int_{B_{R}}|X w|^{2} d x+c_{\varepsilon} \int_{B_{R}}|X u|^{2} d x+c_{\varepsilon} \int_{B_{R}}|X \psi|^{2} d x
\end{aligned}
$$

Choosing $\varepsilon<\lambda$ leads to

$$
\int_{B_{R}}|X w|^{2} d x \leq c \int_{B_{R}}|X u|^{2} d x+c \int_{B_{R}}|X \psi|^{2} d x
$$

On the basis of (4.2), it follows from Lemma 3.3 that for any $0<\rho \leq R$

$$
\begin{aligned}
& \int_{B_{\rho}}|X u|^{2} d x \\
& \quad \leq 2 \int_{B_{\rho}}|X w|^{2} d x+2 \int_{B_{\rho}}|X(u-w)|^{2} d x \\
& \quad \leq c\left[\left(\frac{\rho}{R}\right)^{Q}+\varepsilon+\eta_{R}(A)\right] \int_{B_{R}}|X w|^{2} d x+c \int_{B_{R}}|X \psi|^{2} d x+2 \int_{B_{\rho}}|X(u-w)|^{2} d x
\end{aligned}
$$




$$
\begin{aligned}
\leq & c\left[\left(\frac{\rho}{R}\right)^{Q}+\varepsilon+\eta_{R}(A)\right] \int_{B_{R}}|X u|^{2} d x \\
& +c \int_{B_{R}}|X \psi|^{2} d x+2 \int_{B_{R}}|X(u-w)|^{2} d x .
\end{aligned}
$$

Note that $w-u$ is admissible as a test function in the definition of weak solutions to the obstacle problem due to $w-u \in S_{X, 0}^{1,2}\left(B_{R}, \mathbb{R}^{N}\right)$ and $w \geq \psi$ a.e. in $B_{R}$. Applying $w-u$ to (1.2) leads to

$$
\int_{\Omega} A(x) X u \cdot X(u-w) d x \leq \int_{\Omega} B(x, u, X u)(u-w) d x+\int_{\Omega} g(x, u, X u) \cdot X(u-w) d x .
$$

From (H1)-(H2) and Hölder's inequality, we have

$$
\begin{aligned}
& \lambda \int_{B_{R}}|X u-X w|^{2} d x \\
& \leq \int_{B_{R}} A(x) X(u-w) \cdot X(u-w) d x \\
& \leq-\int_{B_{R}} A(x) X w \cdot X(u-w) d x+\int_{B_{R}}|B(x, u, X u)||u-w| d x \\
&+\int_{B_{R}}|g(x, u, X u)||X u-X w| d x \\
& \leq-\int_{B_{R}} A(x) X \psi \cdot X(u-w) d x+\int_{B_{R}}\left(|f|+L|X u|^{\gamma_{0}}\right)|u-w| d x \\
&+\int_{B_{R}}\left(|\tilde{f}|+L|X u|^{\gamma}\right)|X u-X w| d x \\
& \leq c\left(\int_{B_{R}}|X u-X w|^{2} d x\right)^{\frac{1}{2}}\left[\left(\int_{B_{R}}|X \psi|^{2} d x\right)^{\frac{1}{2}}+\left(\int_{B_{R}}\left(|f|+|X u|^{\gamma_{0}}\right)^{2 Q /(Q+2)} d x\right)^{\frac{Q+2}{2 Q}}\right] \\
&+c\left(\int_{B_{R}}|X u-X w|^{2} d x\right)^{\frac{1}{2}}\left(\int_{B_{R}}\left(|\tilde{f}|+|X u|^{\gamma}\right)^{2} d x\right)^{\frac{1}{2}},
\end{aligned}
$$

which means

$$
\begin{aligned}
\int_{B_{R}}|X u-X w|^{2} d x \leq & c \int_{B_{R}}|X \psi|^{2} d x+c \int_{B_{R}}\left(|\tilde{f}|+|X u|^{\gamma}\right)^{2} d x \\
& +c\left(\int_{B_{R}}\left(|f|+|X u|^{\gamma_{0}}\right)^{2 Q /(Q+2)} d x\right)^{\frac{Q+2}{Q}} .
\end{aligned}
$$

In view of $1 \leq \gamma_{0}<\frac{Q+2}{Q}, 0 \leq \gamma<1$, it follows by Hölder's inequality that

$$
\begin{aligned}
\left(\int_{B_{R}}|X u|^{2 \gamma_{0} Q /(Q+2)} d x\right)^{\frac{Q+2}{Q}} & =\left[\left(\int_{B_{R}}|X u|^{2 \gamma_{0} Q /(Q+2)} d x\right)^{\frac{Q+2}{2 \gamma_{0} Q}}\right]^{2 \gamma_{0}} \\
& \leq\left[\left|B_{R}\right|^{\frac{Q+2}{2 \gamma_{0} Q}-\frac{1}{2}}\left(\int_{B_{R}}|X u|^{2} d x\right)^{\frac{1}{2}}\right]^{2 \gamma_{0}} \\
& =\left|B_{R}\right|^{\frac{Q+2}{Q}-\gamma_{0}}\left(\int_{B_{R}}|X u|^{2} d x\right)^{\gamma_{0}}
\end{aligned}
$$


and

$$
\begin{aligned}
\int_{B_{R}}|X u|^{2 \gamma} d x & \leq\left[\left|B_{R}\right|^{\frac{1}{2 \gamma}-\frac{1}{2}}\left(\int_{B_{R}}|X u|^{2} d x\right)^{\frac{1}{2}}\right]^{2 \gamma} \\
& \leq\left|B_{R}\right|^{1-\gamma}\left(\int_{B_{R}}|X u|^{2} d x\right)^{\gamma} \\
& \leq \varepsilon \int_{B_{R}}|X u|^{2} d x+c_{\varepsilon}\left|B_{R}\right| .
\end{aligned}
$$

Combine (4.4)-(4.6) to deduce

$$
\begin{aligned}
\int_{B_{R}}|X u-X w|^{2} d x \leq & c(\omega(R)+\varepsilon) \int_{B_{R}}|X u|^{2} d x+c \int_{B_{R}}|X \psi|^{2} d x \\
& +c\left(\int_{B_{R}}|f|^{2 Q /(Q+2)} d x\right)^{\frac{Q+2}{Q}}+c \int_{B_{R}}|\tilde{f}|^{2} d x+c_{\varepsilon}\left|B_{R}\right|,
\end{aligned}
$$

where $\omega(R)=\left|B_{R}\right|^{\frac{Q+2}{Q}-\gamma_{0}}\left(\int_{B_{R}}|X u|^{2} d x\right)^{\gamma_{0}-1}$.

From (4.7) and (4.3), we find, for any $0<\rho \leq R$ (we may suppose $R<1$ ),

$$
\begin{aligned}
\int_{B_{\rho}}|X u|^{2} d x \leq & c\left[\left(\frac{\rho}{R}\right)^{Q}+\varepsilon+\eta_{R}(A)+\omega(R)\right] \int_{B_{R}}|X u|^{2} d x+c \int_{B_{R}}|X \psi|^{2} d x \\
& +c\left(\int_{B_{R}}|f|^{2 Q /(Q+2)} d x\right)^{(Q+2) / Q}+c \int_{B_{R}}|\tilde{f}|^{2} d x+c\left|B_{R}\right| \\
\leq & c\left[\left(\frac{\rho}{R}\right)^{Q}+\vartheta(R)\right] \int_{B_{R}}|X u|^{2} d x+\tilde{c} \frac{\left|B_{R}\right|}{R^{\lambda}},
\end{aligned}
$$

where $\vartheta(R)=\varepsilon+\eta_{R}(A)+\omega(R), \tilde{c}=\tilde{c}\left(\|f\|_{L^{2 Q /(Q+2), \lambda Q /(Q+2)}}^{2}+\|\tilde{f}\|_{L^{2, \lambda}}^{2}+\|X \psi\|_{L^{p, \lambda}}^{2}\right)$. By the absolute continuity of Lebesgue integral, we know that $\omega(R) \rightarrow 0$ as $R \rightarrow 0$. Finally, we can take $R<$ $R_{0}$ such that $\eta_{R}(A)$ is small enough due to the VMO property of $A(x)$. If we take $F(\rho)=\frac{\left|B_{\rho}\right|}{\rho^{\lambda}}$, $0<Q-\lambda<\tau<Q$, we claim that $\frac{\rho^{\tau}}{F(\rho)}=\frac{\rho^{\tau+\lambda}}{\left|B_{\rho}\right|}$ is almost increasing. In fact, it follows from (2.2) that, for any $t \in(0,1)$,

$$
\frac{(t \rho)^{\tau}}{F(t \rho)} / \frac{\rho^{\tau}}{F(\rho)}=\frac{(t \rho)^{\tau+\lambda}}{\left|B_{t \rho}\right|} / \frac{\rho^{\tau+\lambda}}{\left|B_{\rho}\right|}=\frac{t^{\tau+\lambda}\left|B_{\rho}\right|}{\left|B_{t \rho}\right|} \leq C_{d}^{2} t^{\tau+\lambda-Q} \leq C_{d}^{2}
$$

By Lemma 4.1, we obtain, for $0<\rho \leq R$,

$$
\int_{B_{\rho}}|X u|^{2} d x \leq c \frac{\left|B_{\rho}\right|}{\rho^{\lambda}}
$$

which shows that $X u \in L_{X, \text { loc }}^{2, \lambda}\left(\Omega, \mathbb{R}^{m N}\right)$.

On the other hand, from Poincaré inequality and (4.8) we see that

$$
\int_{B_{\rho}}\left|u-u_{\rho}\right|^{2} d x \leq c \rho^{2} \int_{B_{\rho}}|X u|^{2} d x \leq c \frac{\left|B_{\rho}\right|}{\rho^{\lambda-2}},
$$


which implies $u \in \mathcal{L}_{X, \text { loc }}^{2, \lambda-2}\left(\Omega, \mathbb{R}^{N}\right)$ and so $u \in C_{X}^{0,(2-\lambda) / 2}\left(\Omega, \mathbb{R}^{N}\right)$ according to Lemma 2.5. The proof is finished.

\section{Acknowledgements}

This work was supported by the National Natural Science Foundation of China (No. 11201258), National Science Foundation of Shandong Province of China (ZR2011AM008, ZR2011AQ006, ZR2012AM010).

\section{Competing interests}

The authors declare that they have no competing interests.

\section{Authors' contributions}

Both authors contributed equally to the writing of this paper. Both authors read and approved the final manuscript.

\section{Publisher's Note}

Springer Nature remains neutral with regard to jurisdictional claims in published maps and institutional affiliations.

\section{Received: 13 December 2017 Accepted: 23 February 2018 Published online: 05 March 2018}

\section{References}

1. Meyers, N.G.: An $L^{p}$-estimate for the gradient of solutions of second order elliptic divergence equations. Ann. Sc. Norm. Super. Pisa, Sci. Fis. Mat., III. Ser. 17, 189-206 (1963)

2. Campanato, S.: Equazioni ellittiche del II ${ }^{\circ}$ ordine e spazi $\mathcal{L}^{(2, \lambda)}$. Ann. Mat. Pura Appl. 69(4), $321-381$ (1965). https://doi.org/10.1007/BF02414377

3. Campanato, S.: Sistemi ellittici in forma divergenza. Regolarita all'interno. Quaderni. Pisa, Scuola Normale Superiore (1980)

4. Campanato, S.: Hölder continuity of the solutions of some nonlinear elliptic systems. Adv. Math. 48, 15-43 (1983). https://doi.org/10.1016/0001-8708(83)90003-8

5. Agmon, S., Douglis, A., Nirenberg, L.: Estimates near the boundary for solutions of elliptic partial differential equations satisfying general boundary conditions. I. Commun. Pure Appl. Math. 12, 623-727 (1959). https://doi.org/10.1002/cpa.3160120405

6. Agmon, S., Douglis, A., Nirenberg, L.: Estimates near the boundary for solutions of elliptic partial differential equations satisfying general boundary conditions. II. Commun. Pure Appl. Math. 17, 35-92 (1964). https://doi.org/10.1002/cpa.3160170104

7. Daněček, J.: Regularity for nonlinear elliptic systems of second order. Comment. Math. Univ. Carol. 27, 755-764 (1986)

8. Giaquinta, M.: Multiple integrals in the calculus of variations and nonlinear elliptic systems. Annals of Mathematics Studies, vol. 105. Princeton University Press, Princeton (1983)

9. Huang, Q.: Estimates on the generalized Morrey spaces $L_{\varphi}^{2, \lambda}$ and $\mathrm{BMO}_{\psi}$ for linear elliptic systems. Indiana Univ. Math. J. 45(2), 397-439 (1996). https://doi.org/10.1512/iumj.1996.45.1968

10. Daněček, J., Viszus, E.: A note on regularity for nonlinear elliptic systems. Arch. Math., Brno 36(3), 229-237 (2000)

11. Daněček, J., Viszus, E.: $\mathcal{L}^{2, \Phi}$ regularity for nonlinear elliptic systems of second order. Electron. J. Differ. Equ. 2002,20 (2002). doiurl10.1007/0-306-47096-9_3

12. Zheng, S., Feng, Z:: Regularity for quasi-linear elliptic systems with discontinuous coefficients. Dyn. Partial Differ. Equ. 5(1), 87-99 (2008). https://doi.org/10.4310/DPDE.2008.v5.n1.a4

13. Gao, D., Niu, P., Wang, J.: Partial regularity for degenerate subelliptic systems associated with Hörmander's vector fields. Nonlinear Anal., Theory Methods Appl. 73(10), 3209-3223 (2010). https://doi.org/10.1016/..na.2010.07.001

14. Dong, Y., Niu, P.: Regularity for weak solutions to nondiagonal quasilinear degenerate elliptic systems. J. Funct. Anal. 270(7), 2383-2414 (2016). https://doi.org/10.1016/j.jfa.2016.02.006

15. Di Fazio, G., Fanciullo, M.S.: Gradient estimates for elliptic systems in Carnot-Carathéodory spaces. Comment. Math. Univ. Carol. 43(4), 605-618 (2002)

16. Di Fazio, G., Fanciullo, M.S.: BMO regularity for elliptic systems in Carnot-Carathéodory spaces. Commun. Appl. Nonlinear Anal. 10(2), 81-95 (2003)

17. Zheng, S., Feng, Z.: Regularity of subelliptic $p$-harmonic systems with subcritical growth in Carnot group. J. Differ. Equ 258(7), 2471-2494 (2015). https://doi.org/10.1016/j.jde.2014.12.020

18. Giaquinta, M.: Remarks on the regularity of weak solutions to some variational inequalities. Math. Z. 177, 15-31 (1981). https://doi.org/10.1007/BF01214336

19. $\mathrm{Mu}$, J., Ziemer, W.P.: Smooth regularity of solutions of double obstacle problems involving degenerate elliptic equations. Commun. Partial Differ. Equ. 16(4-5), 821-843 (1991). https://doi.org/10.1080/03605309108820780

20. Choe, H.J.: A regularity theory for a general class of quasilinear elliptic partial differential equations and obstacle problems. Arch. Ration. Mech. Anal. 114(4), 383-394 (1991). https://doi.org/10.1007/BF00376141

21. Choe, H.J.: Regularity for certain degenerate elliptic double obstacle problems. J. Math. Anal. Appl. 169(1), 111-126 (1992). https://doi.org/10.1016/0022-247X(92)90106-N

22. Marchi, S.: Regularity for the solutions of double obstacle problems involving nonlinear elliptic operators on the Heisenberg group. Matematiche 56(1), 109-127 (2001)

23. Duzaar, F., Gastel, A.: Nonlinear elliptic systems with Dini continuous coefficients. Arch. Math. 78(1), 58-73 (2002). https://doi.org/10.1007/s00013-002-8217-1

24. Wang, J., Liao, D.: Optimal partial regularity for nonlinear sub-elliptic systems related to Hörmander's vector fields. Kyushu J. Math. 65(2), 251-277 (2011). https://doi.org/10.2206/kyushujm.65.251

25. Wang, J., Liao, D., Gao, S., Yu, Z.: Optimal partial regularity for sub-elliptic systems with Dini continuous coefficients under the superquadratic natural growth. Nonlinear Anal. 114,13-25 (2015). https://doi.org/10.1016/j.na.2014.10.028 
26. Chen, S., Tan, Z.: Optimal partial regularity for nonlinear sub-elliptic systems. J. Math. Anal. Appl. 387(1), 166-180 (2012). https://doi.org/10.1016/j.jmaa.2011.08.080

27. Qiu, Y.: Optimal partial regularity of second order nonlinear elliptic systems with Dini continuous coefficients for the superquadratic case. Nonlinear Anal. 75(8), 3574-3590 (2012). https://doi.org/10.1016/j.na.2012.01.016

28. Bögelein, V., Duzaar, F., Habermann, J., Scheven, C.: Partial Hölder continuity for discontinuous elliptic problems with VMO-coefficients. Proc. Lond. Math. Soc. 103(3), 371-404 (2011). https://doi.org/10.1112/plms/pdr009

29. Tan, Z., Wang, Y., Chen, S.: Partial regularity in the interior for discontinuous inhomogeneous elliptic system with VMO-coefficients. Ann. Mat. Pura Appl. 196(1), 85-105 (2017). https://doi.org/10.1007/s10231-016-0564-9

30. Simon, L.: Lectures on geometric measure theory. Australian National University Press, Canberra (1983)

31. Duzaar, F., Grotowski, J.F.: Optimal interior partial regularity for nonlinear elliptic systems: the method of A-harmonic approximation. Manuscr. Math. 103(3), 267-298 (2000). https://doi.org/10.1007/s002290070007

32. Daněček, J., John, O., Stará, J.: Morrey space regularity for weak solutions of Stokes systems with VMO coefficients. Ann. Mat. Pura Appl. (4) 190(4), 681-701 (2011). https://doi.org/10.1007/s10231-010-0169-7

33. Yu, H., Zheng, S.: Optimal partial regularity for quasilinear elliptic systems with VMO coefficients based on A-harmonic approximations. Electron. J. Differ. Equ. 2015, 16 (2015)

34. Xu, C., Zuily, C.: Higher interior regularity for quasilinear subelliptic systems. Calc. Var. Partial Differ. Equ. 5(4), 323-343 (1997). https://doi.org/10.1007/s005260050069

35. Lu, G.: Embedding theorems on Campanato-Morrey spaces for vector fields of Hörmander type. Approx. Theory Appl. 14(1), 69-80 (1998)

36. Hörmander, L.: Hypoelliptic second order differential equations. Acta Math. 119, 147-171 (1967). https://doi.org/10.1007/BF02392081

37. Nagel, A., Stein, E.M., Wainger, S.: Balls and metrics defined by vector fields. I: basic properties. Acta Math. 155 103-147 (1985). https://doi.org/10.1007/BF02392539

38. Lu, G.: Weighted Poincaré and Sobolev inequalities for vector fields satisfying Hörmander's condition and applications. Rev. Mat. Iberoam. 8(3), 367-439 (1992). https://doi.org/10.4171/RMI/129

39. Hajłasz, P. Koskela, P.: Sobolev met Poincaré. Mem. Am. Math. Soc. 688, 101 (2000)

40. Xu, C.: Subelliptic variational problems. Bull. Soc. Math. Fr. 118(2), 147-169 (1990). https://doi.org/10.24033/bsmf.2141

\section{Submit your manuscript to a SpringerOpen ${ }^{\circ}$ journal and benefit from:}

- Convenient online submission

- Rigorous peer review

- Open access: articles freely available online

- High visibility within the field

- Retaining the copyright to your article

Submit your next manuscript at $\boldsymbol{\nabla}$ springeropen.com 